\title{
Dynamic Load Balancing in Cloud Computing using CloudSim
}

\author{
Jayprakash Maltare \\ M.Tech Scholar \\ Department of Computer \\ Science \& Engineering \\ ,VITM,Indore , India
}

\author{
Balwant Prajapat \\ Assistant Professor \\ Department of Computer \\ Science \& Engineering \\ ,VITM, Indore , India
}

\begin{abstract}
Load balancing is a very important part of cloud computing which makes cloud computing more efficient and ease. Load balancing provides efficient policy to several reviews within cloud computing environment. Comprehensive balancing must get immediately into accounts two tasks. Due to this reason it is easy to implement a scheduler. The reason behind for being simple is that load balancing only mandatory information is a list of nodes, Round Robin FCFS, Equal Load share, Throttled algorithms used for load balancing, Equal load share. Each algorithm has some drawback and proposed algorithm provides effectiveness of load balancing in cloud computing. It uses two data structure one is hash map and another one is list.
\end{abstract}

\section{Keywords}

Cloud computing, load balancing, Task Scheduling, Round Robin, Throttled, Equal Load Sharing, CloudSim

\section{INTRODUCTION}

Through cloud computing the business person can access their personal files at any pc and within the centripetal info storage, method and live knowledge. This technology permits a way to cheaper computing. In cloud model the application resources area unit are shared between personal and public cloud. Cloud computing suppliers have developed tools to share workloads on cloud to manage hybrid conditions, but sometimes they would like all environments to be supported same platform.

For current effective execution cloud programming plays a vital role. Programming refers for the connected set with policies to be able to manage the order of operations. Different individuals are involved fully to programming rule throughout existing distributed system instead job programming is single of them.

The advantage involving programming rule goes to be therefore on accomplishing associate, the simplest methodology production and high performance computing. Programming manages intelligence involving processor memory and smart programming policy provides most utilization of resource.

Cloud Computing has become the foremost viable account the problems that area unit computation intensive. Cloud provides the plan of action of sharing resources and services to user on demand. Virtualized resource and services is employed whereas not the info of geographical variations. Cloud computing provides mechanisms that target run time demand of computing resources, like storage, accessibility, package etc.

The on the market Cloud platforms distinguish among the service kind, the cost, the quality of Service (QoS) what is more as performance. This reality brings Cloud customers the pliability of freely selecting target style from broad varies of Cloud platforms. However at an identical time, this raises the matter of the power among the assorted Clouds [1].

Development of economical service provisioning policies is that the foremost issues in Cloud analysis. Clouds exist in associate open world characterized by constant changes occurring autonomously and unpredictably. Throughout this context, game supposed ways in which modify in-depth analytical understanding of the service provisioning draw back [2]. The cloud computing preponderantly offers three kinds of services Infrastructure as a Service (IaaS), Platform as a Service (PaaS), and package as a Service (SaaS).

The various objectives of improvement criteria is [2]:

- Central processing unit Utilization: The overall proportion of your time that CPU was used or used i.e. wasn't idle.

- Throughput: Total no. of tasks dead (or requests served) per unit time.

- Response Time: The time spent by missive of invitation within the waiting queue until it gets the primary time to use the central processing unit.

- Waiting Time: the overall time spent by the request waiting within the prepared queue once the primary response from central processing unit.

- Turnaround Time: the overall time taken by missive of invitation to induce fully served, together with its latency, waiting time and repair time.

- Fairness: The principle that states that each request ought to get equal share of central processing unit time.

- Resource Cost: The overall price of the resources noninheritable or used for the coupling of requests by varied cloud customers. The goal is to maximize the central processing unit Utilization, maximize the outturn, minimize the latency, minimize the Waiting time, minimize the work time, minimize the Resource price and adapt the Fairness principle. 


\section{LITERATURE REVIEW}

In the gift situation, mobile cloud atmosphere with the work is split and distributed in little jobs that have constant size of Cloudlets. These Cloudlets virtual machine further as varied programming policies area unit determined in accordance with FCFS, Round Robin etc. CloudComputinglandscape operate users usually gift/show ar e killed.

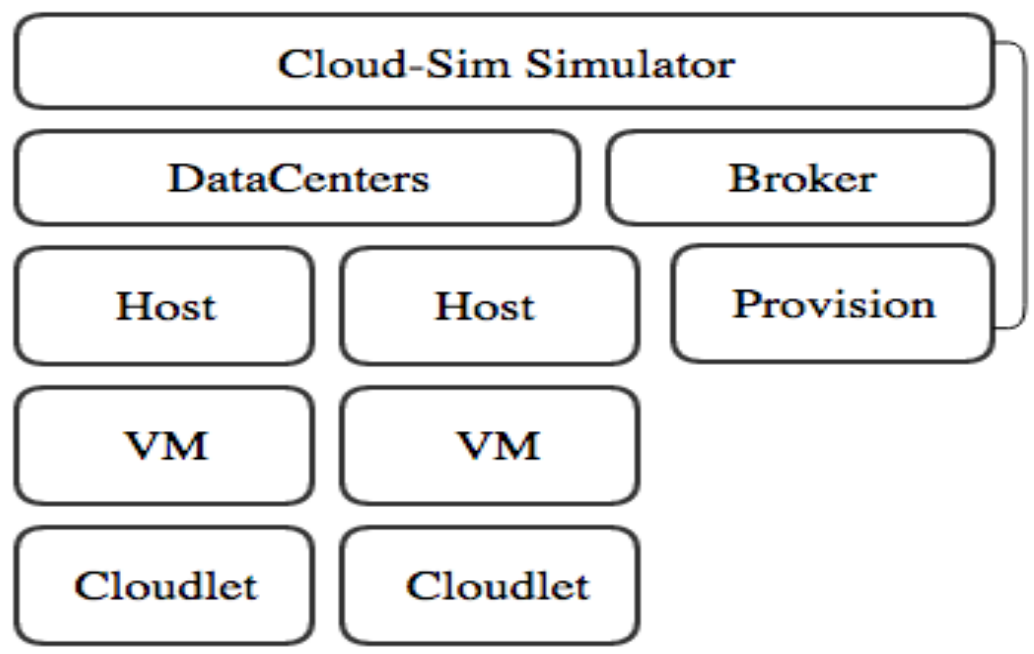

Figure1. Block diagram of Components of Cloud System

Cloud organizer (CC) [2] equal in size cloudlets divides and datacenter (DC). Normally pass FCFS manner because it is extremely time overwhelming as a result of cloudlets Associate in Nursing action at a time and once they reach VM . VM cloudlets enforced in line as they reach the VM. In fact, it's very long task default scheduled policy value is insensitive and inefficient.

The letter [7] could be a latest VM manned filling equalization algorithmic program is weighted equalization algorithmic program very active police investigation, so as to urge higher nonetheless the datacenter CloudSim realize tools to expeditiously facilitate individuals incontestable weight distribution virtual instruments performance standards for load equalization requests. VMS works with the powers related to completely different process / requests area unit sometimes issued on the VM named or even all-powerful and is that the lowest since thus on.

In the paper [8] The author planned that a random hymenopter us insect colony improvement algorithmic program for optimizing virtual machine approach is sometimes associated with the allocation of jobs on your current incoming be obtained.

\section{Components Of Cloud System}

A typical implementation of cloud modeling involves four establishments CloudSim Datacenters, Host, in conjunction with system software system additionally to the applying kind virtual $\mathrm{M} / \mathrm{C}$ that is shown in figure1.

1. Datacenter: Set of rules called datacenters. This virtual model (VMS) (for example, could also be accountable in addressing VM provisioning.

2. Datacenter Broker: VM provisioning VMs submitted requests to submit assignments on the system for information centers and systems to be in a position people: it modifies one or two of mechanisms.
3. Host: a decent host is connected to the information center. Ideas will host virtual machines.

4. VM: this is often a machine that the applying virtual machine (VM), that is a physical machine, known as the enforced software system implementation represents. Every virtual machine operating operations by the host to pay their own resources.

5. Cloudlet: category programming policy which will be enforced at intervals the datacenter through a broker category is employed.

\section{LOAD BALANCING ALGORITHMS}

System dynamics square measure vital, reckoning on load leveling schemes will be either static or dynamic. Here could be a dynamic theme is employed for its flexibility.

Some exit load leveling algorithms are:

Few exiting load-balancing algorithms are as follows:

1. Token Routing

2. Round Robin

3. Randomized

4. Central Queue

5. Throttled

6. Equal load sharing

Throttled and equal load sharing algorithms.

Throttled : Throttled: technique one a listing of all VMs for every row of someone relies on the method of maintaining indexes to early starts. Size and availableness of the machine on the thought of a match is found, the load balancer receives missive of invitation of the buyer which the buyer allocates VM. So, however the factors that have a load balancer VM accessible Match-1 and so the request is queued come. 


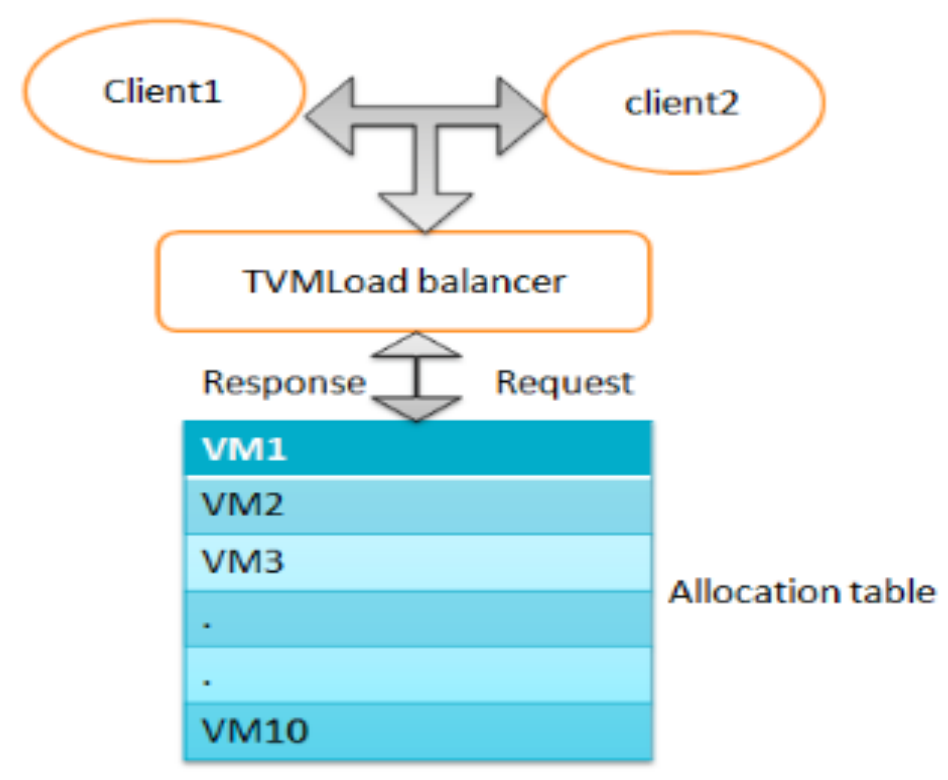

Figure2. Block diagram of Throttled Algorithm

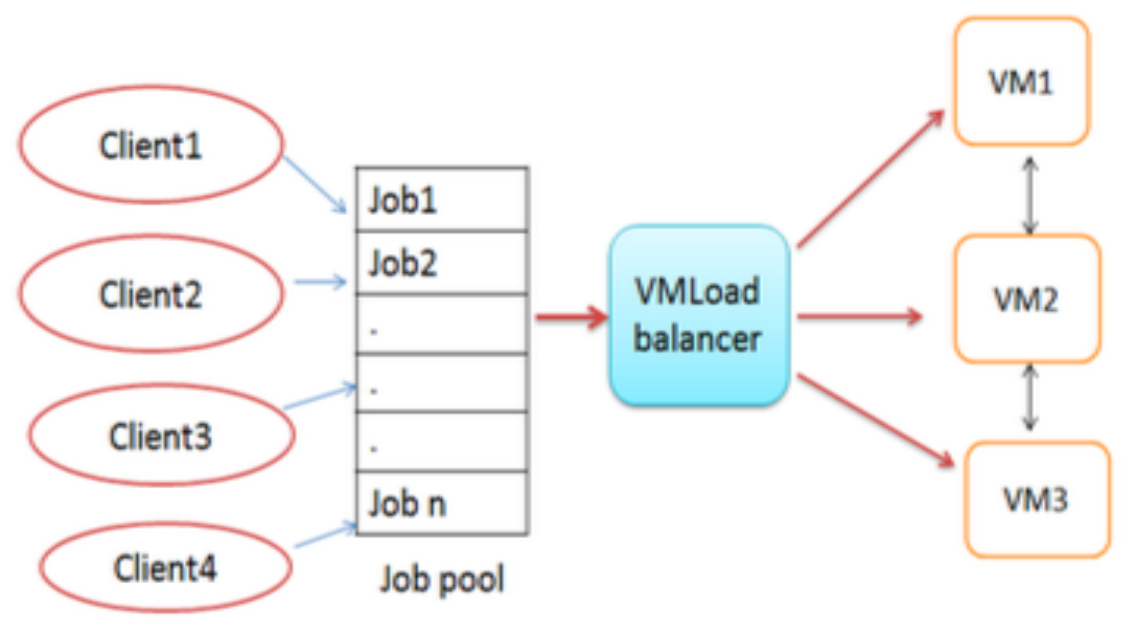

Figure3. Block diagram of Equal load sharing Algorithm

Equally Load Share : Equally Load Share: The algorithmic rule checks the scale of the rule and so the primary technique could be a virtual machine that's loaded gently distributes the load by transferring hundreds indiscriminately. The load on the load balancer nodes utterly completely different spreads, and so, unfold it's spoken as spectrum techniques. Load Balancer roles presently arrest and needed to use the services of the virtual machine maintains a queue of pattern. Athlete therefore ceaselessly the queue and so scans the list of virtual machines.

\section{PROBLEM IN EXISTING SYSTEM}

Load balancing schemes counting on whether or not the system dynamics area unit $\mathrm{v}$ may be either static or dynamic. Static schemes don't use the system data and area unit less advanced. 


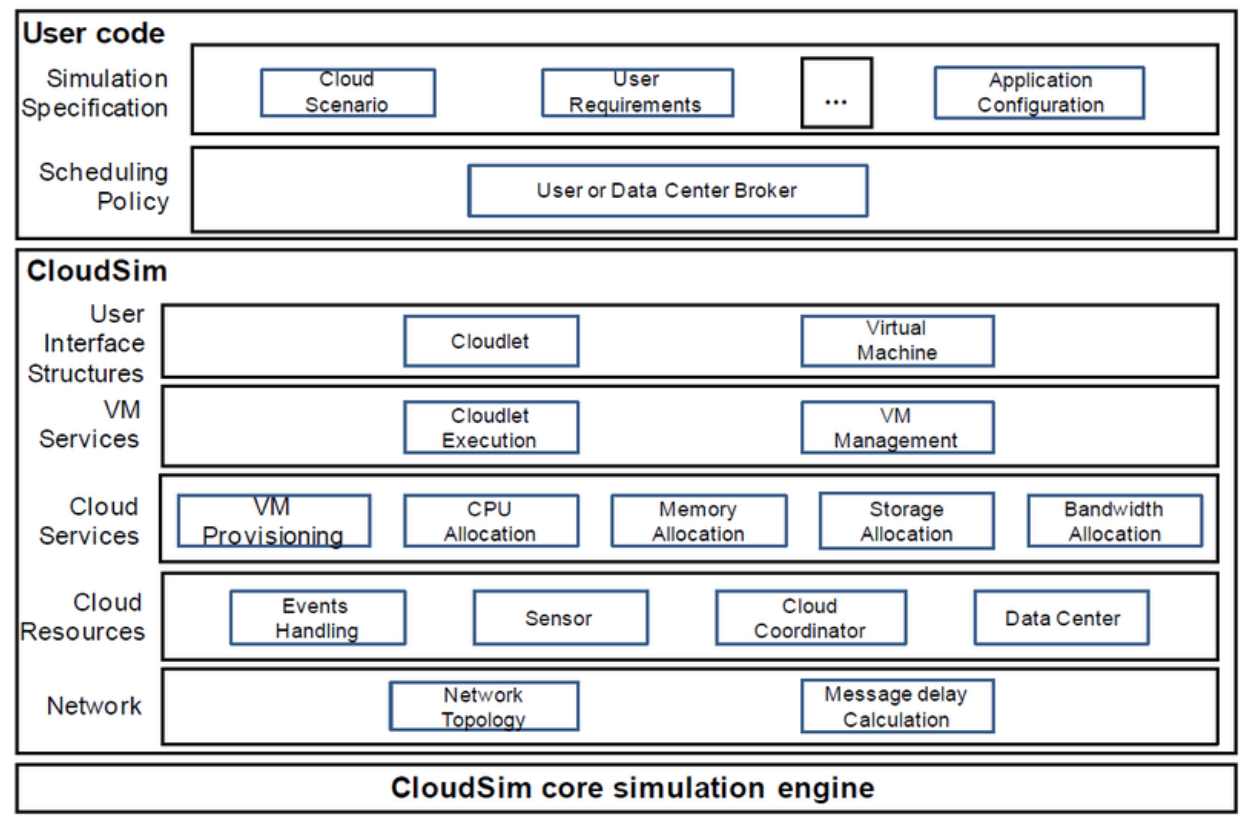

Fig 4. CloudSim Architecture

Considering that the work arrival pattern is not predictable and also the capacities of every one node in dissent, for load managing downside, work management is very important to boost system performance and keep stability. A dynamic theme may be used here for its freedom.

- Cloud-computing surroundings may be a awfully advanced downside together with load equalization receiving.

- The job arrival pattern is not predictable and also the capacities of every one node within the dissent, for load managing downside, work management is very important to boost system performance and keep stability.

Load balancing schemes counting on whether or not the system dynamics area unit $\mathrm{v}$ may be either static or dynamic. Static schemes don't use the system data and area unit less advanced.

Considering that the work arrival pattern is not predictable and also the capacities of every one node in dissent, for load managing downside, work

\section{CLOUDSIM SIMULATOR}

CloudSim [12] is that the several economical tool you'll be able to use with respect to modeling relating to Cloud. throughout your current lifecycle of associate degree Cloud, CloudSim permits VMs for you to be managed coming back from hosts that may within flip area unit sometimes managed by datacenters.

CloudSim offers design within four uncomplicated entities. These kinds of entities supply shopper to set-up the basic cloud computing surroundings yet as live your effectiveness involving replenish equalization algorithms.. Datacenters entity options the responsibility of providing Infrastructure level solutions for the Cloud Users. They act as a home to assist a ton of Host Entities or even a ton of instances hosts' entities mixture to assist application type the solitary Datacenter entity. Hosts with Cloud area unit sometimes Physical Servers the thought have pre-configured process capabilities. Host is really accountable relating to providing software system level SERVICE towards Cloud Users. Hosts have their explicit storage and memory. process options relating to hosts is sometimes expressed throughout MIPS per second.

\section{PROPOSED ALGORITHM}

The proposed algorithm improvement over the Round Robin VM Load Balancing algorithm. The Round Robin algorithmic program doesn't save the state of previous allocation of a VM to a call for participation from a given user base whereas a similar state is saved in RR VM load balancer. The Round Robin VM Load balancer maintain two data structure which is discussed below. 


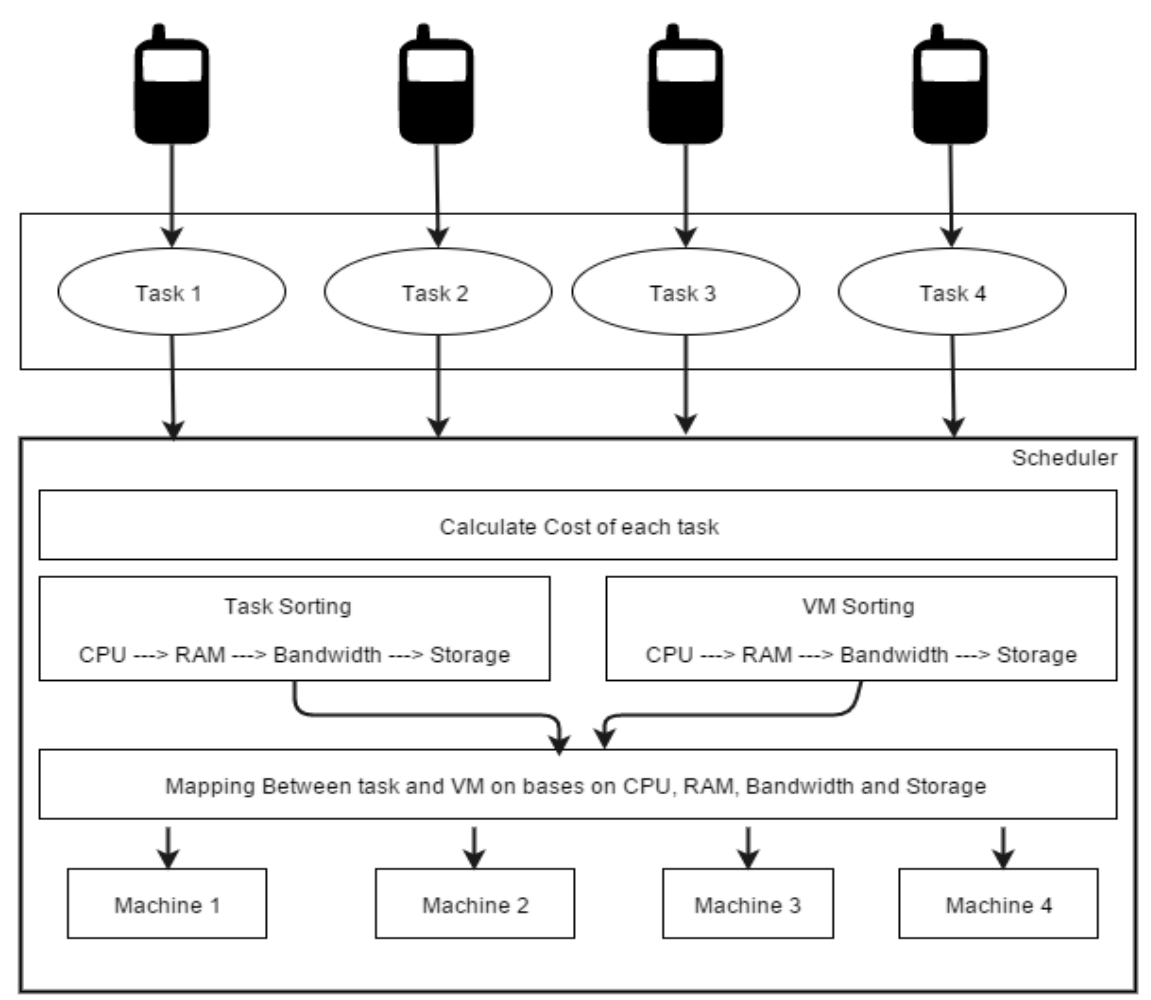

Fig 5. CloudSim Architecture

Hash Map- In which it stores the entry for the last VM allocated to a request from a given user base.

VM State List- this stores the allocation status (i.e. busy available) of each VM.

Proposed System performs the following steps:

- Calculate the cost of each task Sort the task according the following parameters

- $\mathrm{CPU}$

- RAM

- Bandwidth

- $\quad$ Storage

- Also arrange the VM according the following parameters

- $\mathrm{CPU}$

- RAM

- $\quad$ Bandwidth

- Storage

- Check the status of Each VM.

- Schedule the sorted VMs on the basis of sorted task

- Calculate throughput, response time of each task

\section{RESULT}

Proposed system implement in java language with cloud sim simulator. Created cloudlets, VMs, Datacenters and Host with specific configuration. After create cloudlets request for VM on Datacenter. We also design resources allocation policy for CloudSim simulator. Resources Allocation policy describe how resources allocated to cloudlets. Configuration parameters show in below table.
Table 1. CloudSim configuration

\begin{tabular}{|c|c|}
\hline \multicolumn{2}{|c|}{ Simulation Parameters } \\
\hline Parameters & Values \\
\hline $\begin{array}{c}\text { Number of } \\
\text { users }\end{array}$ & 6 \\
\hline $\begin{array}{c}\text { Number of } \\
\text { VMs }\end{array}$ & 4 \\
\hline $\begin{array}{c}\text { Number of } \\
\text { Cloudlets }\end{array}$ & 40 \\
\hline
\end{tabular}

\begin{tabular}{|c|c|}
\hline \multicolumn{2}{|c|}{ Cloudlets Details } \\
\hline Parameters & Values \\
\hline $\begin{array}{l}\text { Cloudlets } \\
\text { Length }\end{array}$ & 50000 \\
\hline File Size & 400 \\
\hline Output Size & 400 \\
\hline PES & 2 \\
\hline
\end{tabular}

\begin{tabular}{|c|c|}
\hline \multicolumn{2}{|c|}{ VM Details } \\
\hline Parameters & Values \\
\hline Name of VM & Xen-VM \\
\hline RAM & 512 \\
\hline MIPS & 250 \\
\hline BW & 1000 \\
\hline
\end{tabular}

\begin{tabular}{|c|c|}
\hline \multicolumn{2}{|c|}{ Datacenter Details } \\
\hline Parameters & Values \\
\hline $\begin{array}{c}\text { Number of } \\
\text { Datacenters }\end{array}$ & 2 \\
\hline MIPS & 1000 \\
\hline RAM & 16384 \\
\hline Storage & 1000000 \\
\hline
\end{tabular}




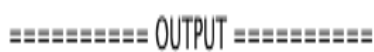

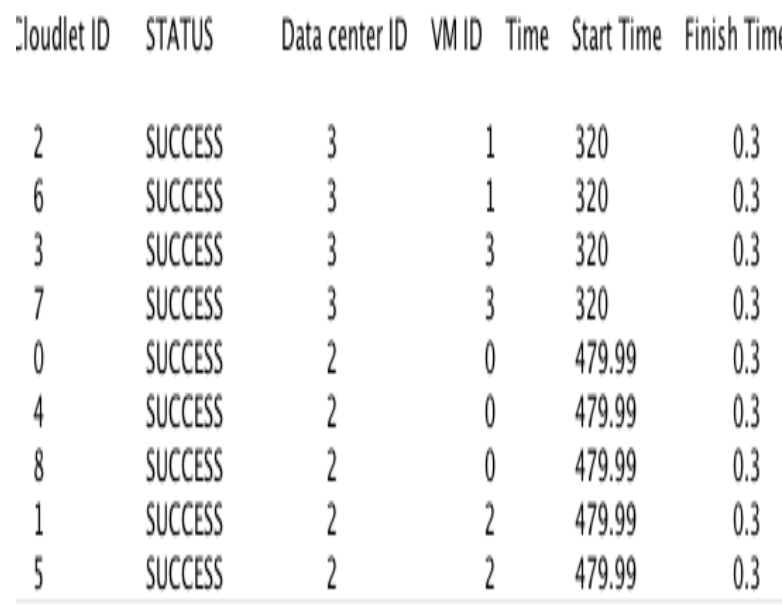

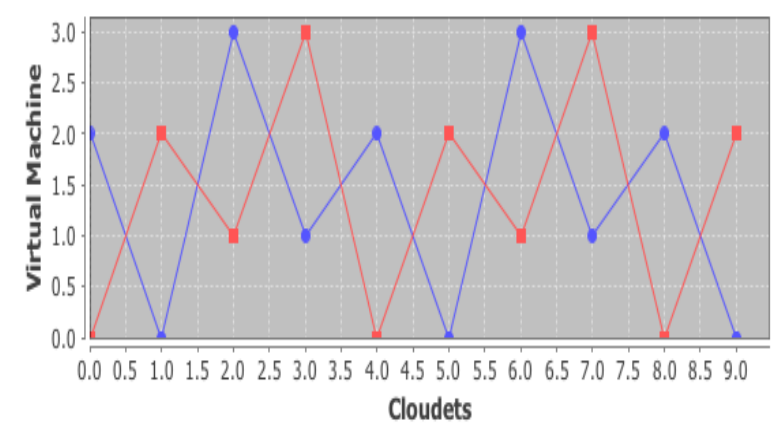

- Existing Algo VM ID $\rightarrow$ Proposed Algo VM ID

Fig 6. VM allocation using existing and proposed System

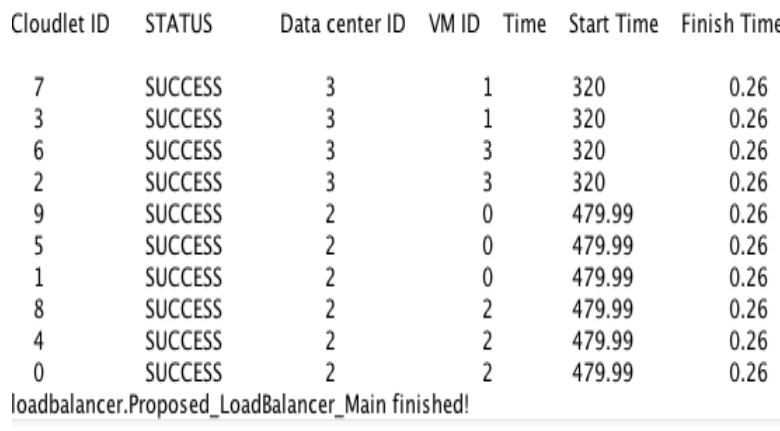

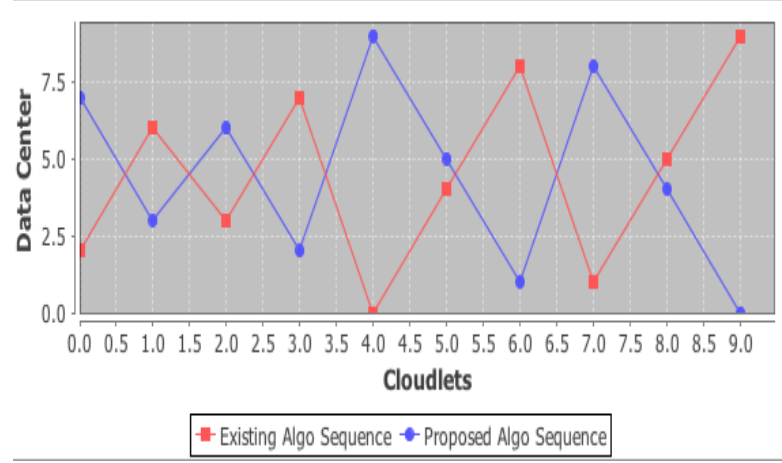

Fig 7. Datacenter allocation using existing and proposed System

\section{CONCLUSION}

This paper presents a thought of Cloud Computing together with analysis challenges in load equalization. It additionally specialize in deserves and demerits of the cloud computing. Major thrust is given on the study of load equalization algorithmic rule, followed by a comparative survey of those abovementioned algorithms in cloud computing with reference to stability, resource utilization, static or dynamicity, cooperative or non-cooperativeness and method migration. This paper aims towards the institution of performance chemical analysis on existing VM load equalization algorithmic rule then enforced in CloudSim and java language

\section{REFERENCES}

[1] Ritu Kapur "A Cost Effective approach for Resource Scheduling in Cloud Computing" IEEE International Conference on Computer, Communication and Control (IC4-2015).

[2] Aditya Makwe, Priyesh Kanungo"Scheduling in Cloud Computing Environment Using Analytic Hierarchy Process Model" IEEE International Conference on Computer, Communication and Control (IC4-2015).

[3] Kunal Mahurkar1, Shraddha Katore2 and Suraj Bhaisade3, Pratikawale4, "Reducing Cost of Provisioning in Cloud Computing", International Journal of Advance in Computer Science and Cloud Computing, Volume- 1, Issue- 2, nov.- 2013, pg. 6- 8.

[4] Dr. Rakesh Rathi1, Vaishali Sharma2 and Sumit Kumar Bole3, "Round Robin Data Center Selection in Single Region for Service Proximity Service Broker in Cloud Analyst" , International Journal of Computer \& Technology, Volume 4 no. 2, March- April 2013, pg. no. 254- 260.

[5] Bhatiya Wickremansinghe1, Rodrigo N. Calheiros2and Dr. Rajkumar Buyya3, "CloudAnalyst: A CloudSimbased Visul Modeller for Analysing Cloud Computing Environments and Applications", IEEE Computer Society, 2010, pp. 446-452.

[6] Pooja Samall and Pranati Mishra2, "Analysis of Variants in Round Robin Algorithms for Load Balancing in Cloud Computing", (IJCSIT) International Journals of Computer Science and Information Technologies, Volume 4 (3), 2013, pg. no. 416- 419.

[7] Kunal Mahurkar1, Shraddha Katore2 and Suraj Bhaisade3, Pratikawale4, "Reducing Cost of Provisioning in Cloud Computing", International Journal of Advance in Computer Science and Cloud Computing, Volume- 1, Issue- 2, nov.- 2013, pg. 6- 8 .

[8] Dr. Rakesh Rathi1, Vaishali Sharma2 and Sumit Kumar Bole3, "Round Robin Data Center Selection in Single Region for Service Proximity Service Broker in Cloud Analyst" , International Journal of Computer \& Technology, Volume 4 no. 2, March- April 2013, pg. no. 254- 260.

[9] Bhatiya Wickremansinghe1, Rodrigo N. Calheiros2and Dr. Rajkumar Buyya3, "CloudAnalyst: A CloudSimbased Visul Modeller for Analysing Cloud Computing Environments and Applications", IEEE Computer Society, 2010, pp. 446-452. 
[10] Jaspreet Kaur, "Comparison of load balancing algorithm in a Cloud", International Journal of Engineering Research and Applications (IJERA), vol. 2, Issue 3, May- June 2012, pp. 1169- 1173

[11] Syed Tauhid Zuheri1, Tamanna Shamrin2 and Rusia Tanbin3, Firoj Mahmud4, "An Efficient Load Balancing Approach in Cloud Environment by using Round Robin Algorithm", International Journal of Artificial and Mechatronics, volume 1, issue 5, 2013, pp 96-99.

[12] B. Santosh Kumar1 and Dr. Latha Parthiban2, "An Implementation of Load Balancing Policy for Virtual Machines Associated with a Data Centre", International Journal of Computer Science \& Engineering Technology (IJCSET), volume 5 no. 03, March 2014, pp. 253- 261.

[13] Sonika Matele1, Dr, K James2 and Navneet Singh3, "A Study of Load Balancing Issue Among Multifarious Issues of Cloud Computing Environment", International Journals of Emerging Technolog Computational and Applied Science (IJETCAS), volume 13- 142, 2013, pg. 236- 241.
[14] Subasish Mohapatra1, Subhadarshini2 and K. Smruti Rekha3, "Analysis of Different Varients in Round Robin Algorithms for Load Balancing in Cloud Computing", International Journal of Computer Application, Volume 69- no. 22, may 2013, pp. 17-21.

[15] Dr Hemant S. Mahalle1, Prof Parag R. Kaver2 and Dr. Vinay Chavan3, "Load Balancing on Cloud Data Centres", Internatinal Journal of Advanced Reserch in Computer Science and Software Engineering, volume 3, issue 1, January 2013, pp. 1- 4.

[16] Randles1, M Lamb2 and Taleb Bendiab3, "A Comparative Studyinto Distributed Load Balancing Algorithm for Cloud Computing", Advanced Information Networking and Application Workshop (WAINA) 2010.

[17] Dr. Rajkumar Buyya, "CloudSim: a toolkit for modelling and simulation of cloud computing environments and evaluation of resource provisioning algorithm", published online 24 august in Wiley Online Library 2010, pp. 23- 50 